\title{
OCORRÊNCIA DE PROCESSOS FONOLÓGICOS EM CRIANÇAS RESIDENTES EM ACOLHIMENTO INSTITUCIONAL
}

\author{
PHONOLOGICAL PROCESSES OCCUR IN CHILDREN RESIDENT IN \\ INSTITUTIONAL CARE
}

\author{
Shoyama Nadja da Silva Franco e Santos ${ }^{1}$ \\ Ivonaldo Leidson Barbosa Lima ${ }^{2}$ \\ Brunna Thaís Luckwu de Lucena ${ }^{3}$ \\ Maria Edvany de Melo Pereira ${ }^{4}$ \\ Veridiana Rosa da Silva ${ }^{5}$ \\ Eva Carolina Fonseca de Rezende Cruz ${ }^{6}$
}

\begin{abstract}
RESUMO: Caracteriza-se alteração de fala a produção inadequada dos sons, uso inadequado das regras fonológicas da língua como também a relação à distribuição do som e ao tipo de sílaba. Para que a criança adquira as bases para um desenvolvimento saudável da linguagem faz-se necessário a interação com a família e um ambiente estimulador, com isso a privação precoce e institucionalização afetam o desenvolvimento emocional, cognitivo, social e neurofisiológico da mesma. Nesse sentido, o objetivo deste estudo foi investigar a ocorrência de processos fonológicos em crianças residentes em acolhimento institucional. A pesquisa foi realizada em um acolhimento institucional com a participação de dez crianças com faixa etária entre quatro e onze anos de idade e oito cuidadores/responsáveis do acolhimento. Foram utilizados dois questionários elaborados pelas pesquisadoras direcionado ao público já referido e, por conseguinte, foi feita avaliação fonológica mediante a utilização do protocolo ABFW - Teste de Fonologia com as crianças. Os dados foram tabulados para posterior análise estatística descritiva e inferencial. Os processos fonológicos encontrados sem maior frequência foram simplificação de líquida, simplificação do encontro consonantal e simplificação de consoante final. Em relação ao grau da alteração fonológica em sua maioria ocorreu no sexo masculino, até nove anos de idade, com até 21 meses de institucionalização, apresentando prevalência no grau leve na etapa de imitação e moderado leve na etapa de nomeação. Pode-se concluir que a maioria das crianças institucionalizadas apresentaram diversos processos fonológicos em seu discurso, sendo necessária uma avaliação e terapia fonoaudiológica mais direcionada.
\end{abstract}

\section{PALAVRAS-CHAVE:}

Fonoaudiologia. Criança Institucionalizada. Desenvolvimento Fonológico. Processos Fonológicos.

\begin{abstract}
Speech disorders is characterized by inadequate production of sounds, inappropriate use of phonological rules of the language, as well as the distribution of sound and syllable type. For the child to acquire the basis for a healthy development of language, it is necessary to interact with the family and a stimulating environment, thus the early deprivation and institutionalization affect the emotional, cognitive, social and neurophysiological development of the same. In this sense, the aim of this study was to investigate the phonological processes occur in children living in institutional care. The research was conducted in an institutional care with the participation of ten children aged between four and eleven years and eight caregivers / guardians of the host. We used two questionnaires prepared by the researchers addressed to the aforementioned public and, therefore, phonological evaluation was made using the protocol ABFW - Phonology Test with
\end{abstract}

\footnotetext{
${ }^{1}$ Pós-graduanda em Linguagem pelo Instituto de Educação Superior da Paraíba (IESP). E-mail: shoyamafranco@gmail.com

${ }^{2}$ Mestre em Linguística pela Universidade Federal da Paraíba (UFPB). E-mail: ivonaldoleidson@gmail.com ${ }^{3}$ Mestre em Modelos de Decisão em Saúde pela Universidade Federal da Paraíba (UFPB). E-mail: brunnaluckwu@hotmail.com

${ }^{4}$ Mestre em Educação para o Ensino na área de Saúde pela Faculdade Pernambucana de Saúde (FPS). E-mail: edy.melop@gmail.com

${ }^{5}$ Pós-graduanda em Fonoaudiologia Hospitalar e Disfagia pelo Centro Universitário de João Pessoa (UNIPÊ). Email: veridianarosa14@gmail.com

${ }^{6}$ Mestre em Gerontologia pela Universidade Federal da Paraíba (UFPB). E-mail: eva.cruz@unipe.edu.br
} 
Volume 14 - Número 1 - mai/ago de 2019

children. Data were tabulated for later descriptive and inferential statistical analysis. The most frequent phonological processes found were liquid simplification, consonant cluster simplification and final consonant simplification. Regarding the degree of phonological disorders, it occurred mostly in males, up to nine years old, with up to 21 months of institutionalization, presenting prevalence in mild degree in imitation stage and moderate mild in naming stage. It can be concluded that most institutionalized children presented several phonological processes in their discourse, requiring a more targeted assessment and speech-language therapy.

KEYWORDS: Speech-Language and Hearing Sciences. Institutionalized Child. Phonological Development. Phonological Processes.

\section{Introdução}

O desenvolvimento fonológico é um processo complexo e ocorre diferentemente entre as crianças. No desenvolvimento fonológico típico, as habilidades auditivas e fonoarticulatórias se desenvolvem e a criança vai paulatinamente superando as dificuldades pertencentes ao desenvolvimento e agregando fonemas no seu inventário fonológico, sendo possível observar na medida em que a idade avança (CERON; SOARES, 2017).

No processo de desenvolvimento da fala, a criança adquire o inventário fonético e organiza os sons de acordo com as regras linguísticas da língua de origem. A criança experimenta diversos processos fonológicos na tentativa de aproximar a sua produção de fala à do adulto, para que, aproximadamente aos quatro anos, já tenha condições de produzir e utilizá-las adequadamente todos os sons da língua materna (PRATES; MARTINS, 2011).

A partir da interação com a família e um ambiente estimulador, a criança adquire as bases para um desenvolvimento saudável da linguagem, no que diz respeito à sua forma, conteúdo e uso, uma vez que os primeiros anos de vida da criança são determinantes para o desenvolvimento apropriado da linguagem. Fatores como o contexto social, familiar e histórico pré, peri e pós-natal do indivíduo como também suas experiências, capacidades cognitivas e orgânico-funcionais são de extrema relevância no processo de aquisição normal da linguagem (PRATES; MARTINS, 2011).

Desse modo, pode-se caracterizar a alteração de fala pela produção inadequada dos sons, uso inadequado das regras fonológicas da língua como também a relação à distribuição do som e ao tipo de sílaba, que resultam em bloqueios fonêmicos, comprometendo o significado da mensagem. Para o diagnóstico do transtorno fonológico se faz necessário analisar as estruturas silábicas presentes na amostra de fala e a distribuição dos sons nessas estruturas, bem como nas palavras, apontando as regras fonológicas que a criança usa e os contrastes que ela mantém em sua fala, além de identificar-se o inventário fonético da mesma (WERTZNER; AMARO; TERAMOTO, 2005).

No que diz respeito aos aspectos ambientais, a literatura refere que a privação precoce e institucionalização afetam o desenvolvimento emocional, cognitivo, social e neurofisiológico da criança (MISQUIATTI et al., 2015). Nóbrega e Minervino (2011) destacam que o ambiente familiar conhecido e vivenciado pela criança e/ou adolescente é substituído pela instituição que o acolhem, sendo assim, o acolhimento é um momento crítico na vida do referido público. Partindo desse pressuposto, os laços de parentesco da criança institucionalizada passam a se tornar tempestuoso. O elo familiar, de amizade fica comprometido a partir do momento que a criança passa a residir no seu novo "lar".

Portanto, essa pesquisa tem caráter inovador, tornando-se relevante e necessário um estudo aprofundado sobre a temática abordada, ressaltando principalmente a importância do fonoaudiólogo na atuação com crianças institucionalizadas, além de caracterizar o desenvolvimento fonológico das mesmas.

Desta forma, esse estudo teve como objetivo investigar a ocorrência de processos fonológicos em crianças residentes em acolhimento institucional. 


\section{Métodos}

Trata-se de uma pesquisa de campo, quantitativa, observacional, transversal de caráter descritivo, aprovada pelo Comitê de Ética em Pesquisa da Instituição de origem, sob o número 2.829.352.

O local de desenvolvimento desse estudo foi em um acolhimento institucional localizado no município de João Pessoa - PB. No referido acolhimento foram encontradas vinte crianças institucionalizadas, porém, participaram da pesquisa dez crianças com faixa etária entre quatro e onze anos de idade, obedecendo ao critério de inclusão.

Foram incluídas na pesquisa crianças com faixa etária entre quatro e onze anos de idade; naturais da capital onde foi realizada a pesquisa; crianças que não apresentaram comorbidades associadas, como alterações cognitivas e nos órgãos fonoarticulatórios; crianças que responderam o questionário e participaram da etapa de avaliação e por fim, cuidadores/responsáveis do acolhimento que responderam o questionário. Foram excluídas crianças que não completaram a etapa de avaliação e crianças e cuidadores/responsáveis que se recusaram a participar da pesquisa.

Referente aos cuidadores totalizaram dezessete atuantes no acolhimento, contudo, oito participaram do estudo, uma vez que são os responsáveis pelas crianças integrantes. Tratandose de uma amostra censitária para o público mencionado.

Após a aprovação desse estudo pelo Comitê de Ética em Pesquisa, foi apresentado aos cuidadores/responsáveis o TCLE e às crianças participantes da pesquisa, o Termo de Assentimento Livre e Esclarecido do Menor, o qual foi lido de maneira que as mesmas compreendessem facilmente o objetivo do estudo, se fazendo presente nesse momento a coordenadora responsável pela casa de acolhimento, permitindo assim, após as assinaturas dos mesmos, a realização da coleta dos dados. O estudo obedeceu às normas e diretrizes regulamentadas pela resolução 466/12 da Comissão de Ética em Pesquisa/CONEP, que regulamenta as pesquisas envolvendo seres humanos e respeitou as normas da Lei n. ${ }^{\circ}$ 8.069, de 13 de julho de 1990 do Estatuto da Criança e do Adolescente - ECA.

A coleta de dados foi dividida em duas etapas, inicialmente foram utilizados dois questionários elaborados pelas pesquisadoras, sendo: o primeiro direcionado aos cuidadores/responsáveis do acolhimento institucional, contendo doze perguntas, sendo nove objetivas e três subjetivas; e o segundo para as crianças, contendo nove perguntas, sendo sete objetivas e duas subjetivas.

O questionário para os cuidadores/responsáveis foi composto por doze perguntas, sendo essas referentes a: dados e informações sobre os cuidadores, seguido por perguntas sobre a sua rotina no acolhimento institucional e sua relação com as crianças abrigadas, questionamentos sobre o desenvolvimento global e possíveis alterações apresentadas pelas crianças, motivo pelo qual a criança foi encaminhada ao abrigo e, por fim, questionamentos sobre o conhecimento geral acerca da Fonoaudiologia.

O questionário direcionado para as crianças consistiu em nove perguntas, sendo essas referentes a: dados e informações sobre as mesmas, seguido por perguntas sobre as condições de vida, como era seu cotidiano no ambiente familiar e atualmente no acolhimento institucional e, por fim, questionamentos sobre a relação com os seus cuidadores e se a criança já foi ou ainda é acompanhada por algum atendimento especializado.

Por conseguinte, foi realizada uma avaliação fonológica das crianças mediante o protocolo ABFW - etapa de imitação e nomeação (WERTZNER, 2000), sendo realizada a coleta de amostra de fala mediante a gravação em áudio usando o aplicativo Gravador de Voz Fácil disponível para Android, versão 2.4.5, oferecido por Digipom, para posterior transcrição e análise da mesma. A aplicação do questionário e a avaliação ocorreram em uma sala reservada, garantindo a privacidade do participante. 
A faixa etária do grupo dos cuidadores variou de 27 a 48 anos, com mediana de 41,00 $( \pm 6,557)$ e das crianças de 4 a 11 anos, com mediana de 9,00 $( \pm 2,066)$. Mediante o questionário aplicado aos cuidadores, foi possível coletar informações a respeito de cada criança selecionada para a pesquisa, dos oito entrevistados, dois deles responderam duas vezes, totalizando dez respostas dos cuidadores.

Portanto, 60,0\% $(n=6)$ correspondiam ao sexo feminino e $40,0 \%(n=4)$ ao sexo masculino e todos com a profissão de Educador Social. Quanto ao tempo de atuação dos cuidadores variou de 2 a 120 meses, com mediana de 78,0 ( $\pm 43,562)$.

No que se refere ao motivo do encaminhamento dos menores ao acolhimento institucional, os cuidadores participantes da pesquisa não têm acesso a tal informação, uma vez que corre em segredo de justiça. Foi indagado à coordenação responsável pela casa de acolhimento as justificativas das chegadas das crianças à instituição, porém, não foi possível informar a causa específica de cada criança participante do estudo. Contudo, foi relatado que a razão pelas quais as mesmas se encontram institucionalizadas foi devido a algum tipo de negligência, ou seja, abuso sexual, violência, drogas, alcoolismo, abandono, entre outros.

A análise das provas de fonologia (imitação e nomeação) da ABFW foi realizada com base na amostra de fala coletada mediante a gravação da mesma. Por conseguinte, foram transcritas as provas e verificada a produtividade dos processos fonológicos, o número de diferentes tipos de processos fonológicos de acordo com a classificação da ABFW (WERTZNER, 2000) e o cálculo do índice PCC-R (Porcentagem de Consoantes Corretas Revisada).

O PCC-R é um dos índices mais utilizados por autores nacionais, em que considera como erros somente as substituições e as omissões e avalia as distorções como acerto. O seu cálculo contabiliza o número total de palavras analisadas na amostra (WERTZNER et al., 2013).

Os dados foram tabulados e analisados a partir de planilhas no Software Microsoft Office Excel ${ }^{\circledR}$. Os resultados foram obtidos através de análise estatística descritiva e inferencial, por meio do software Statistical Package for Social Sciences (SPSS) versão 20.0 for Windows. Para definição dos testes estatísticos foi realizado o teste de associação Quiquadrado de Pearson (Teste exato de Fisher) não havendo resultado estatisticamente significante $(\mathrm{p}<0,05)$.

\section{Resultados}

- Cuidadores

Tendo em vista que o cuidador é o indivíduo que passa mais tempo na instituição, procurou-se saber como era a sua rotina no acolhimento, $70,0 \%(n=7)$ dos cuidadores responderam bom de acordo com suas experiências e tempo de atuação na atual instituição. Averiguou-se saber dos participantes como os mesmos se auto avaliavam, sendo verificado que grande parte dos cuidadores 70,0\% $(n=7)$ responderam bom. Quando questionado o nível de afetividade entre os cuidadores e as crianças, observa-se que 50,0\% ( $n=5)$ dos entrevistados afirmaram ter um bom relacionamento (Tabela 1).

Tabela 1: Distribuição de variáveis categorizadas

\begin{tabular}{lccc}
\hline \multicolumn{1}{c}{ Questões } & \multicolumn{2}{c}{ Percepção dos Cuidadores } \\
\cline { 2 - 4 } & Excelente & Bom & Regular \\
\hline Rotina do cuidador & $20 \%$ & $70 \%$ & $10 \%$ \\
Auto avaliação do cuidador referente à sua conduta & $10 \%$ & $70 \%$ & $20 \%$ \\
Relação do cuidador com a criança & $40 \%$ & $50 \%$ & $10 \%$ \\
\hline
\end{tabular}

Fonte: Dados do Autor, 2018. 
Dos participantes, $80,0 \%(\mathrm{n}=8)$ afirmaram que acompanham as crianças desde o início do seu acolhimento e apenas $20,0 \%(n=2)$ responderam que não.

Buscou-se saber dos entrevistados se as crianças que se encontram institucionalizadas apresentam algum tipo de dificuldade, $60,0 \%(\mathrm{n}=6)$ dos cuidadores afirmaram que sim e $40,0 \%(\mathrm{n}=4)$ responderam que não.

Em uma questão subjetiva do questionário, foi indagado aos cuidadores a principal dificuldade apresentada pelo menor e estas respostas foram categorizadas. O maior percentual observado foi no quesito que diz respeito às dificuldades de comportamento da criança, que representa 30,0\% $(n=3)$ da população pesquisada e 20,0\% $(n=2)$ dos participantes afirmaram problemas na fala (Tabela 2 ).

Tabela 2: Percepção do cuidador quanto às alterações das crianças institucionalizadas

\begin{tabular}{lcc}
\hline Tipo de dificuldade & Frequência & Porcentagem (\%) \\
\hline Dificuldades de comportamento & 3 & 30,0 \\
Problemas na fala & 2 & 20,0 \\
Dificuldade de aprendizagem & 1 & 10,0 \\
Não apresenta alteração & 4 & 40,0 \\
\hline Total & $\mathbf{1 0}$ & $\mathbf{1 0 0 , 0}$ \\
\hline
\end{tabular}

Fonte: Dados do Autor, 2018.

Pesquisou-se a percepção dos cuidadores quanto ao comportamento das crianças no aspecto social, psicológico e emocional. No quesito social 50,0\% $(n=5)$ das crianças apresentaram ter um bom relacionamento com as demais crianças e cuidadores. Quanto ao aspecto psicológico, os participantes responderam que $30,0 \%(\mathrm{n}=3)$ das crianças são tranquilas. Pertinente ao aspecto emocional foi observado que 40,0\% ( $n=4)$ das crianças apresentaram comportamento normal e 40,0\% $(n=4)$ feliz (Tabela 3$)$.

Tabela 3: Percepção do cuidador referente ao comportamento das crianças institucionalizadas

\begin{tabular}{lcc}
\hline Aspectos & Frequência & Porcentagem (\%) \\
\hline
\end{tabular}

Aspecto social

$\begin{array}{lll}\text { Bom relacionamento com as demais crianças e cuidadores } & 5 & 50,0\end{array}$

$\begin{array}{lll}\text { Tranquila } & 1 & 10,0\end{array}$

$\begin{array}{lll}\text { Comportamento bom } & 2 & 20,0\end{array}$

$\begin{array}{lll}\text { Isolamento social } & 1 & 10,0\end{array}$

$\begin{array}{lll}\text { Autoritária } & 1 & 10,0\end{array}$

\section{Aspecto psicológico}

Tranquila $\quad 3 \quad 30,0$

$\begin{array}{lll}\text { Feliz } & 2 & 20,0\end{array}$

Resistência/Rejeição $\quad 1 \quad 10,0$

$\begin{array}{lll}\text { Estressada } & 1 & 10,0\end{array}$

Abalada $\quad 1 \quad 10,0$

\section{Aspecto emocional}

\begin{tabular}{lll} 
Normal & 4 & 40,0 \\
Feliz & 4 & 40,0 \\
Agitada & 1 & 10,0 \\
Carente & 1 & 10,0 \\
\hline
\end{tabular}

Fonte: Dados do Autor, 2018. 


\section{- Crianças}

Referente ao tempo de institucionalização das crianças, as respostas foram quantificadas em meses, que variou de 2 a 28, com mediana de 21,0 $( \pm 10,698)$.

Realizou-se um levantamento com as crianças para saber se as mesmas gostavam dos seus cuidadores e, em seguida, sendo caracterizado o nível de afetividade para com eles. Em referência ao primeiro questionamento, equivaleu a $90,0 \%(n=9)$ para sim e $10,0 \%(n=1)$ para não. Posteriormente, foi averiguado que $90,0 \%(n=9)$ da amostra afirmaram ter uma boa relação com os cuidadores, enquanto $10,0 \%(n=1)$ classificou como ruim.

Optou-se por analisar as alterações de fala considerando as características fonológicas, em que 100,0 (n=10) apresentaram simplificação de líquida, 70,0\% (n=7) simplificação do encontro consonantal e 50,00\% (n=5) simplificação de consoante final (Tabela 4). Ressalta-se que as categorias não são excludentes, ou seja, a mesma criança poderia apresentar mais de um processo fonológico.

Tabela 4: Distribuição dos processos fonológicos

\begin{tabular}{lcc}
\hline Processos Fonológicos & Frequência & Porcentagem (\%) \\
\hline Redução de sílaba & 3 & 30 \\
Harmonia consonantal & 1 & 10 \\
Plosivização de fricativas & 1 & 10 \\
Posteriorização para velar & 1 & 10 \\
Posteriorização para palatal & 2 & 20 \\
Frontalização de palatal & 2 & 20 \\
Simplificação de líquidas & 10 & 100 \\
Simplificação do encontro & 7 & 70 \\
Consonantal & 5 & 50 \\
Simplificação da consoante final & 1 & 10 \\
Ensurdecimento de fricativas & 10 & \\
\hline
\end{tabular}

Fonte: Dados do Autor, 2018

Pertinente ao PCC-R etapa de imitação o valor mínimo observado foi 85,9 e máximo de 96,2 , com mediana de $89,650( \pm 3,5982)$. Enquanto a etapa de nomeação variou de 61,1 a 96,6 , com mediana de $81,100( \pm 10,4930)$.

A tabela 5 apresenta os resultados em que foi realizado o teste de associação Quiquadrado de Pearson (Teste exato de Fisher) não havendo resultado estatisticamente significante $(\mathrm{p}<0,05)$.

Tabela 5: Distribuição de variáveis categorizadas e sua relação com o grau da alteração fonológica

\begin{tabular}{|c|c|c|c|c|c|c|}
\hline \multirow[b]{2}{*}{$\begin{array}{l}\text { Grau da alteração } \\
\text { fonológico }\end{array}$} & \multicolumn{2}{|c|}{ Sexo } & \multicolumn{2}{|c|}{ Idade } & \multicolumn{2}{|c|}{ Tempo de Institucionalização } \\
\hline & Feminino & Masculino & $\begin{array}{c}\text { Até } \\
9 \\
\text { anos }\end{array}$ & $\begin{array}{c}\text { Acima } \\
\text { de } \\
9 \text { anos }\end{array}$ & $\begin{array}{l}\text { Até } 21 \\
\text { meses }\end{array}$ & $\begin{array}{l}\text { Acima de } 21 \\
\text { meses }\end{array}$ \\
\hline & $\begin{array}{c}N \\
(\%)\end{array}$ & $\begin{array}{c}\mathrm{N} \\
(\%) \\
\end{array}$ & $\begin{array}{c}\mathrm{N} \\
(\%)\end{array}$ & $\begin{array}{c}\mathbf{N} \\
(\%)\end{array}$ & $\begin{array}{c}\mathrm{N} \\
(\%)\end{array}$ & $\begin{array}{c}\mathrm{N} \\
(\%)\end{array}$ \\
\hline \multicolumn{7}{|l|}{ Imitação } \\
\hline Leve & 3 & 6 & 6 & 3 & 5 & 4 \\
\hline & $100,0 \%$ & $85,7 \%$ & $85,7 \%$ & $100,0 \%$ & $83,3 \%$ & $100,0 \%$ \\
\hline \multirow[t]{2}{*}{ Moderado Leve } & 0 & 1 & 1 & 0 & 1 & 0 \\
\hline & $0,0 \%$ & $14,3 \%$ & $14,3 \%$ & $0,0 \%$ & $16,7 \%$ & $0,0 \%$ \\
\hline \multirow[t]{2}{*}{ Moderado grave } & 0 & 0 & 0 & 0 & 0 & 0 \\
\hline & $0,0 \%$ & $0,0 \%$ & $0,0 \%$ & $0,0 \%$ & $0,0 \%$ & $0,0 \%$ \\
\hline \multirow[t]{2}{*}{ Grave } & 0 & 0 & 0 & 0 & 0 & 0 \\
\hline & $0,0 \%$ & $0,0 \%$ & $0,0 \%$ & $0,0 \%$ & $0,0 \%$ & $0,0 \%$ \\
\hline
\end{tabular}




\begin{tabular}{rrrrcccc}
\hline Nomeação & Leve & 0 & 1 & 0 & 1 & 1 & 0 \\
& $0,0 \%$ & $14,3 \%$ & $0,0 \%$ & $33,3 \%$ & $16,7 \%$ & $0,0 \%$ \\
& & 5 & 5 & 2 & 4 & 3 \\
Moderado Leve & 2 & $66,7 \%$ & $71,4 \%$ & $71,4 \%$ & $66,7 \%$ & $66,7 \%$ & $75,0 \%$ \\
& 0 & 1 & 1 & 0 & 0 & 1 \\
Moderado grave & $0,0 \%$ & $14,3 \%$ & $14,3 \%$ & $0,0 \%$ & $0,0 \%$ & $25,0 \%$ \\
& 1 & 0 & 1 & 0 & 1 & 0 \\
Grave & 1 & $0,0 \%$ & $14,3 \%$ & $0,0 \%$ & $16,7 \%$ & $0,0 \%$ \\
\hline
\end{tabular}

Teste de comparação de Fisher: p>0,05; Fonte: Dados do Autor, 2018.

\section{Discussão}

Os indivíduos que convivem e crescem em ambientes saudáveis e incentivadores tendem a apresentar um desenvolvimento aprimorado das suas capacidades, sendo assim a limitação e a ausência desses estímulos na infância e na adolescência pode resultar em maiores dificuldades de desenvolvimento no futuro, visto que durante todas as etapas da vida do ser humano o desenvolvimento é cumulativo (FERREIRA, 2014).

A principal fonte de cuidado e proteção às crianças é a família, contudo devido a diversos motivos algumas famílias demonstram dificuldade em desempenhar seu papel de proteção, apoio e cuidado aos filhos. Diante os principais riscos no contexto familiar para o bom desenvolvimento infantil destacam-se alcoolismo e droga por parte dos responsáveis, abuso sexual, violência doméstica, abandono e negligência (LAUZ; BORGES, 2013). Concernente ao motivo pelo qual a criança foi acolhida não foram concebíveis tais informações para o presente estudo, visto que corre em segredo de justiça. Porém, a coordenação da instituição pesquisada informou que todas as crianças foram encaminhadas por algum tipo de negligência. Tais motivos impactam diretamente na qualidade de vida da criança (NAKATOMI et al., 2018).

De acordo com os dados obtidos na pesquisa, investigou-se saber como era a rotina do cuidador no acolhimento, sua autoavaliação e nível de afetividade com as crianças para assim entender melhor o vínculo cuidador e criança. Os resultados em sua maioria foram bons para os quesitos mencionados. Estudo corrobora que as interações do cuidador com as crianças podem melhorar os resultados comportamentais e condições institucionais do menor (NAKATOMI et al., 2018). A vivência no ambiente institucional proporciona a criação de laços afetivos significativos, mesmo na ausência da figura materna e paterna (OLIVEIRA; PRÓCHNO, 2010).

Segundo Ferreira (2014) não há dúvidas que ocorre uma quebra da família a partir do momento em que o indivíduo passa a residir na casa de acolhimento, por mais que seja momentânea tal situação poderá acarretar diversos impactos no seu desenvolvimento. Em relação à existência de algum tipo de dificuldade apresentada pelas crianças acolhidas ao saber do cuidador, foi constatado que $60,0 \%$ afirmaram presença de dificuldade e $40,0 \%$ responderam não. Visto que, $30,0 \%$ da amostra correspondeu a dificuldades de comportamento da criança.

Em referência à percepção dos cuidadores quanto ao comportamento das crianças no aspecto social, psicológico e emocional, foi observado que a maioria das respostas correspondeu a um bom relacionamento com as demais crianças e cuidadores. A literatura refere que ao contrário do que se supõe à primeira vista, as percepções das crianças não são tão problemáticas, uma vez que os cuidadores e as outras crianças apresentam relações com certo nível de afetividade (OLIVEIRA; PRÓCHNO, 2010).

No que tange o tempo em que as crianças forem restritas do convívio familiar e o tempo de espera, tais situações interferem não somente na acomodação em caso de retorno aos seus familiares de origem, mas também nos casos de inclusão permanente em outra parentela, dessa maneira as consequências de uma temporada de institucionalização serão maiores segundo a idade da criança no instante em que essa for distanciada da família (NÓBREGA; MINERVINO, 2011), com isso estudo confirma que a inserção precoce no 
âmbito familiar das crianças institucionalizadas também trará benefícios em seu desenvolvimento (BOS et al., 2011). Na referida pesquisa, no que diz respeito ao tempo de institucionalização das crianças, as respostas foram quantificadas em meses, que variou de 2 a 28, com mediana de 21,0.

Fatores determinantes como restrição social, estresse, limitação ao acesso de promoção à saúde, exposições ambientais, entre outros, influencia nas condições biológicas individuais, apresentando relação com o surgimento e prevalência das alterações fonoaudiológicas, tal como, alterações de linguagem, que poderá ser afetado pelo ambiente em que se vive (CEBALLOS; CARDOSO, 2009).

\section{Considerações finais}

Dentre os processos fonológicos os mais frequentes foram simplificação de líquida, simplificação do encontro consonantal e simplificação de consoante final. Em relação ao grau da alteração fonológica em sua maioria ocorreu no sexo masculino, até nove anos de idade, com até 21 meses de institucionalização, apresentando prevalência no grau leve na etapa de imitação e moderado leve na etapa de nomeação.

Os dados sinalizam a importância de uma intervenção fonoaudiológica mais direcionada a esse público, a fim de garantir uma avaliação adequada dos fatores etiológicos dessas alterações e das manifestações de linguagem apresentadas, possibilitando o desenvolvimento de estratégias terapêuticas sensíveis a cada caso.

Espera-se que os resultados da presente pesquisa possam alertar e sensibilizar os profissionais de saúde sobre a importância de um olhar mais criterioso sobre as alterações de fala e suas implicações no desenvolvimento de crianças acolhidas, favorecendo às mesmas um benéfico padrão de comunicação e melhor qualidade de vida no âmbito social, visto que é um tema pouco explorado na literatura.

\section{Referências}

BOS, K. Psychiatric outcomes in young children with a history of institutionalization. Harv Rev Psychiatry. v. 19, n. 1, p. 15-24. 2011.

CEBALLOS, A. G. C.; CARDOSO, C. Determinantes sociais de alterações fonoaudiológicas. Revista Soc Bras Fonoaudiol, Salvador, v. 14, n. 3, p. 441-5. 2009.

CERON, M.I.; SOARES, M.K. Desenvolvimento fonológico. In: LAMÔNICA, D.A.C; BRITO, D.B.O. (Orgs.). Tratado de Linguagem: perspectivas contemporâneas. São Paulo: Book Toy. 2017.

FERREIRA, F. P. M. Crianças e adolescentes em abrigos: uma regionalização para Minas Gerais. Revista Serv. Soc. Soc., São Paulo, n. 117, p. 142-168, jan./mar. 2014.

LAUZ, G. V. M.; BORGES, J. L. Concepção de família por parte de crianças em situação de acolhimento institucional e por parte de profissionais. Revista Psicologia Ciência e Profissão. v. 33, n. 4, p. 852-867. 2013.

MISQUIATTI, A.R.N. et al. Desempenho de vocabulário em crianças pré-escolares institucionalizadas. Revista CEFAC, São Paulo, v. 17, n. 3, p. 783-791, maio/jun. 2015.

NAKATOMI, T. et al. Children and adolescents in institutional care versus traditional families: a quality of life comparison in Japan. Health Qual Life Outcomes. v. 16, n. 151. 2018.

NÓBREGA, J.N.; MINERVINO, J.N.N. Análise do nível de desenvolvimento da linguagem em crianças abrigadas. Revista Psicol. Argum, Curitiba, v. 29, n. 65, p. 219-226, abr./jun. 2011. 
OLIVEIRA, S. V. PRÓCHNO, C. C. S. C. A vinculação afetiva para crianças institucionalizadas à espera de adoção. Revista Psicologia Ciência e Profissão. v. 30, n. 1, p. 62-84. 2010.

PRATES, L.P.C.S.; MARTINS, V.O. Distúrbios da fala e da linguagem na infância. Revista Médica, Minas Gerais, v. 21, n. 4. 2011.

WERTZNER H.F.; AMARO, L.; TERAMOTO, S.S. Gravidade do distúrbio fonológico: julgamento perceptivo e porcentagem de consoantes corretas. Pró-Fono Revista de Atualização Científica. v. 17, n. 2, p. 185-194, maio/ago. 2005.

WERTZNER, H. F. Fonologia. In: ANDRADE, C.R.F. et al. (Orgs.). ABFW; teste de linguagem infantil nas áreas de fonologia, vocabulário, fluência e pragmática. Carapicuiba, Pró-Fono. 2000.

WERTZNER, H. F. et al. Aplicação de medidas de gravidade e de inconsistência de fala em crianças com transtorno fonológico. Revista ACR. v. 18, n. 3, p. 213-9. 2013.

Recebido em 18/05/19

Aceito em 30/09/19 